\title{
Change in renin, cardiovascular and inflammatory markers over three years in a black and white population: the SABPA study
}

\author{
Rijané Swart, Johannes M. van Rooyen ${ }^{*}$ and Catharina M. C. Mels
}

\begin{abstract}
Background: To investigate if percentage change $(\% \Delta)$ in renin over a 3 year follow-up is associated with $\% \Delta$ in cardiovascular and inflammatory markers in a low renin bi-ethnic group.

Methods: Blood pressure, active renin, C-reactive protein and interleukin- 6 levels of 73 black and 81 white teachers were measured at baseline and after 3 years.

Results: In the black group, \% $\%$ renin was inversely associated with $\% \Delta$ systolic blood pressure $(\beta=-0.27 ; p=0$. 011). In the white group $\% \Delta$ renin was inversely associated with \% $\Delta$ interleukin-6 ( $\beta=-0.24 ; p=0.005)$.

Conclusions: These prospective results indicate that a decrease in renin over time is associated with an increase in blood pressure in a low renin black South African cohort.
\end{abstract}

Keywords: Race, C-reactive protein, Hypertension, Inflammation, Interleukin-6, Low renin, Systolic blood pressure

\section{Background}

The prevalence of hypertension in South Africa is a major concern. A health survey done in 2001 stated that 8.2 million people above the age of 15 years had hypertension [1]. Furthermore, only 2.7 million people with hypertension receive anti-hypertensive treatment [1]. More recently it was indicated that $78 \%$ of South Africans above 50 years of age have hypertension, underlining the increasing burden of cardiovascular disease (CVD) in South Africa [2].

Increased renin, the rate limiting step in the reninangiotensin system (RAS), is associated with increased synthesis of angiotensin II (Ang II) from angiotensinogen [3]. In turn, increased Ang II levels are associated with increased inflammation [4] and may lead to the development of hypertension [5]. On the other hand, suppressed RAS activity, as a consequence of low renin

\footnotetext{
* Correspondence: Johannes.VanRooyen@nwu.ac.za

Hypertension in Africa Research Team (HART), North-West University

(Potchefstroom Campus), Private Bag X6001, Potchefstroom 2522, South Africa
}

(c) The Author(s). 2017 Open Access This article is distributed under the terms of the Creative Commons Attribution 4.0 International License (http://creativecommons.org/licenses/by/4.0/), which permits unrestricted use, distribution, and reproduction in any medium, provided you give appropriate credit to the original author(s) and the source, provide a link to the Creative Commons license, and indicate if changes were made. The Creative Commons Public Domain Dedication waiver (http://creativecommons.org/publicdomain/zero/1.0/) applies to the data made available in this article, unless otherwise stated. hypertension (low-renin hypertension) [6].

Lower renin levels in black participants when compared to their white counterparts were previously reported [7, 8]. Additionally, we also indicated inverse associations of renin levels with ambulatory systolic and diastolic blood pressure in black men [9]. In low renin black men and women, renin was also associated with the albumin-to-creatinine ratio, suggesting a link between low renin levels and subclinical end-organ damage [10].

In contrast with the lower renin activity found in black participants, levels of inflammation are higher in black participants when compared to white participants [11]. We previously linked increased blood pressure with increased inflammation in a high $\mathrm{C}$-reactive protein (CRP) (>3 mg/L) group of black men [12]. In the present sub-study we therefore aimed to investigate if change (over a three-year period) in renin levels is associated with change in cardiovascular and inflammatory markers in a low renin black and white cohort.

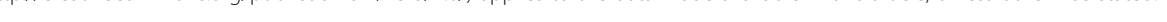




\section{Methods}

\section{Study design and population}

This study formed part of the Sympathetic activity and Ambulatory Blood Pressure in Africans (SABPA) prospective cohort study that took place between 2008/9 (baseline) and 2011/12 (follow-up). A detailed description of the study population and protocol has been published [13]. At baseline 409 black and white teachers with a similar sex distribution were included and 359 teachers returned for the follow-up study $(87.3 \%$ compliance to follow-up). Exclusion criteria at baseline included the use of $\alpha$ - and $\beta$-blockers, an ear temperature $>37.5^{\circ} \mathrm{C}$, participants who were vaccinated or who donated blood 3 months prior to the commencement of the study and pregnant or lactating women. For this sub-study we also excluded participants who did not participate in both the baseline and follow-up phases $(n=50)$ and participants with no renin values at baseline $(n=35)$ and follow-up $(n=68)$. Additionally, in accordance with our aims participants with a renin value of $\geq 6.18 \mathrm{pg} / \mathrm{ml}$ at baseline $(n=88)$ and follow-up $(n=17)$ was excluded in this study. The cutoff value of $6.18 \mathrm{pg} / \mathrm{ml}$ was selected based on the mean renin value in healthy subjects between 40 and 60 years of age (supine range:1.1-20.2 pg/ml) (Renin III Generation, CIS Biointernational, Cedex, France) [10]. HIV infected participants $(n=13)$ and participants on medication $(n=1)$ that could interfere with the RAS was also excluded. After applying these exclusion criteria our baseline study population consisted of 73 black and 81 white participants while data from 66 black and 71 white participants were used to investigate change over a 3 year period.

\section{Anthropometric measurements}

Weight and height of the subjects were taken in triplicate with calibrated instruments (Precision Health Scale, A \& D Company, Tokyo, Japan and Invicta Stadiometer, IP 1465, UK) and body mass index was calculated [14].

\section{Questionnaires}

All the subjects completed a general health questionnaire containing questions on their lifestyle habits such as smoking and medication usage.

\section{Cardiovascular measurements}

The validated Finometer device $[15,16]$ was used to measure systolic blood pressure (SBP) and diastolic blood pressure (DBP) after $5 \mathrm{~min}$ resting period (Finapres Medical Systems, Amsterdam, The Netherlands). Total peripheral resistance (TPR) and Windkessel compliance (Cwk) were calculated using the Fast Modelflo computer software program from the Finometer data. The Sonosite Micromaxx ultrasound system (SonoSite Inc., Bothell, WA) and a 6-13 $\mathrm{MHz}$ linear array transducer was used to measure carotid intima media thickness (cIMT), by obtaining a high resolution image from two angles from the left and right carotid artery. A $10 \mathrm{~mm}$ segment with good image quality was chosen after the images were imported into the Artery Measurement System (AMS) II v1.139 (Gothenburg, Sweden). The mean cIMT were calculated through determining the borders of the far and near wall and the inner diameter of the vessel from 100 discrete measurements through the $10 \mathrm{~mm}$ segment for each participant. The analysis of the cIMT was done by one observer and the intraobserver variability was $0.04 \mathrm{~mm}$ between two measurements made 4 weeks apart $(n=10)$.

\section{Biochemical analyses}

Experienced nurses obtained blood samples from fasting participants from brachial antecubital vein branches with a sterile winged infusion set. Blood was drawn from participants when in a supine position. Serum, ethylenediaminetetraacetic acid (EDTA) and sodium fluoride plasma were prepared according to standard procedures and stored at $-80{ }^{\circ} \mathrm{C}$ until biochemical analyses were performed. Active renin was determined with a radioimmunometric assay (Renin III Generation, CIS biointernational, Cedex, France) in EDTA plasma. Serum IL-6 levels were measured using a high sensitivity Quantikine enzyme linked immunosorbent assay (ELISA) (R\&D Systems, Minneapolis, MN USA). High sensitivity CRP, gamma glutamyl transferase (GGT) as measure of alcohol abuse [17], triglycerides and total cholesterol levels were determined in serum while glucose levels were determined in sodium fluoride plasma with three multiple analysers (Cobas Integra 400 plus, Roche, Basel, Switzerland; Konelab 20i; Thermo Scientific, Vantaa, Finland and Unicel DXC 800, Beckman and Coulter, Germany). The intra- and inter-coefficients of variation for all assays were below $10 \%$. Von Willebrand Factor (vWf) was determined with an ELISA assay in which a polyclonal rabbit anti-vWf antibody and rabbit antivWf-horse radish peroxidase (HRP) antibody (DAKO, South Africa) were used. Serum cotinine levels were measured using a homogeneous immunoassay (Automated Modular, Roche, Basel, Switzerland).

\section{Statistical analyses}

Statistical analyses were performed with Statistica 12 (Statsoft Inc., Tulsa, OK, USA). Values are regarded as significant when $p \leq 0.05$. Data were expressed as arithmetic mean and standard deviation for normally distributed variables. Variables with a non-Gaussian distribution (renin, IL-6, CRP, vWf, cotinine, GGT) were logarithmically transformed and the central tendency and spread represented by the geometric mean and the 5th and 95th percentile intervals. Means and proportions were compared between black and white groups using independent 
t-tests and Chi-square tests, respectively. Proportions were compared at baseline and follow-up with the McNemar tests, in black and white low renin groups. Percentage change for all variables were calculated and compared between black and white groups while adjusting for age, sex and baseline values using analyses of covariance (ANCOVA). Correlations of percentage change in renin with percentage change in biochemical and cardiovascular variables were performed using partial regression analyses (adjusted for sex and age at baseline). Forward stepwise multiple regression analyses were performed to determine independent associations of percentage change in renin with percentage change in inflammatory and cardiovascular variables. Two models were compiled with percentage change in renin as the dependent variable in both models. In model 1 the main independent variable was percentage change in SBP. The covariates that were entered in model 1 were age, baseline SBP, baseline renin, percentage change in IL-6, baseline IL-6, baseline GGT and percentage change in GGT. We selected covariates for inclusion in our models based on the literature. Age, since blood pressure and vascular change is dependent of age [18], and the black population is known to experience early vascular ageing it was added as covariate to our models. Baseline SBP, baseline renin, baseline IL- 6 and baseline GGT, because we used the percentage change of these variables in the model. Percentage change in IL-6, since our aim was to investigate if change (over a three-year period) in renin levels is associated with change in inflammatory markers thus IL-6. Increased IL-6 may indicate future risk for CVD [19]. Percentage change in GGT, because GGT is a marker of alcohol usage, it may have a strong influence on hypertension and inflammation [20].

In model 2 the main independent variable was percentage change in PP. The covariates entered for model 2 were age, baseline PP, baseline renin, percentage change in IL-6, baseline IL-6, baseline GGT and percentage change in GGT. In a post hoc analyses the achieved power (1- $\beta$ ) were determined for multiple regression models with 8 variables, for a group with a sample size of $n=76$ for blacks $(1-\beta=0.999)$ and $n=99$ for whites $(1-\beta=0.77)$ (G*power v3.1.9.2) [21]. The effect sizes (f2) were determined from the partial regression coefficient values and the significance level $(\alpha)$ was set at 0.05 . In sensitivity analyses, we substituted percentage change in IL-6 with percentage change in CRP and age with BMI in separate analyses. Since anti-inflammatory medication usage may influence the inflammatory response and the results, we also added anti-inflammatory medication usage as covariate and repeated the multiple regression analyses.

\section{Results}

The characteristics of black $(n=73)$ and white $(n=81)$ participants with low renin at baseline are compared in
Table 1. The cardiovascular profile of black and white participants with low renin was comparable, except for the higher DBP $(p=0.02)$ noted in the black group compared to the white group. The number of participants who were hypertensive increased from baseline to follow-up, black group: $n=18(27.3 \%)$ to $n=27$ (40.91\%) $(p=0.087)$ and white group: $n=8(11.27 \%)$ to $n=15(21.13 \%)(p<0.001)$. In both the low renin black and white groups the number of participants using antihypertensive medication increased from baseline to follow-up, black group: $n=0(0.00 \%)$ to $n=12(18.2 \%)$ $(p<0.001)$ and white group: $n=1(1.14 \%)$ to $n=8$ $(11.3 \%)(p<0.001)$.

The black group had lower renin $(3.87 \mathrm{pg} / \mathrm{ml}$ vs. $4.59 \mathrm{pg} / \mathrm{ml} ; p=0.002)$, glucose $(p=0.001)$ and total cholesterol $(p<0.001)$ levels. The inflammatory marker, CRP $(p=0.006)$ as well as vWf levels $(p<0.001)$ were higher in the black participants when compared to their white counterparts. Both cotinine and self-reported smoking were similar between the black and white groups, but the black participants had higher GGT levels $(p<0.001)$ compared to the white participants.

In Table 2, the percentage change in the anthropometric, cardiovascular, lifestyle and biochemical variables are shown. Both SBP and DBP increased in the black group, while it decreased in the white group, with the difference between the groups being significant (SBP: $p=0.02$ and DBP: $p=0.059$ ). Although TPR increased in both groups, the increase in the black group was significantly higher $(p=0.036)$. The cIMT also increased in both racial groups, but to a greater extent in the whites $(p<0.001)$.

Renin decreased in the black group and increased in the white group $(-17.47 \%$ vs. $2.17 \% ; p<0.001)$. Regarding the inflammatory markers, IL-6 increased in both groups but more in the black group (59.44\% vs. $32.51 \%$; $p<0.001$ ) while CRP increased slightly in the black group and decreased in the white group, with the difference between the groups being borderline significant $(p=0.069)$.

In single regression (Fig. 1) and partial regression analyses (Additional file 1: Table S1) percentage change in renin were inversely correlated with percentage change in SBP $(r=-0.30 ; p=0.015)$ (Fig. 1) and with percentage change in PP $(r=-0.36 ; p=0.003)$ (Additional file 1: Table S1) in the low renin black group only. These associations were absent in the low renin white group, however percentage change in renin were inversely correlated with percentage change in IL-6 $(r=-0.25$, $p=0.037$ ), in the low renin white group.

In multiple regression analyses, the inverse association of percentage change in renin with percentage change in SBP in the low renin black group was confirmed to be independent of various covariates $(\beta=-0.27$; $p=0.011$; Table 3). Additionally, percentage change in $\operatorname{GGT}(\beta=0.26 ; p=0.017)$ was also positively 
Table 1 Characteristics of low renin black and white participants at baseline

\begin{tabular}{|c|c|c|c|}
\hline & \multicolumn{3}{|l|}{ Low renin } \\
\hline & Black $(n=73)$ & White $(n=81)$ & $p$-value \\
\hline Age (years) & $41.2 \pm 6.55$ & $46.9 \pm 8.72$ & $<0.001$ \\
\hline Sex ( $n$, male) & 36 & 31 & 0.167 \\
\hline Body mass index $\left(\mathrm{kg} / \mathrm{m}^{2}\right)$ & $28.1 \pm 5.86$ & $27.0 \pm 5.44$ & 0.232 \\
\hline \multicolumn{4}{|l|}{ Cardiovascular measurements } \\
\hline Systolic blood pressure (mmHg) & $136 \pm 16$ & $135 \pm 14$ & 0.793 \\
\hline Diastolic blood pressure (mmHg) & $79 \pm 9$ & $76 \pm 7$ & 0.021 \\
\hline Pulse pressure $(\mathrm{mmHg})$ & $56.4 \pm 10.4$ & $58.9 \pm 10.7$ & 0.141 \\
\hline Total peripheral resistance $(\mathrm{mmHg} / \mathrm{ml} / \mathrm{s})$ & $1.01 \pm 0.25$ & $1.05 \pm 0.3$ & 0.399 \\
\hline Windkessel compliance (ml/mmHg) & $1.91 \pm 0.40$ & $2.02 \pm 0.54$ & 0.148 \\
\hline Carotid intima media thickness (mm) & $0.65 \pm 0.10$ & $0.66 \pm 0.11$ & 0.557 \\
\hline \multicolumn{4}{|l|}{ Biochemical analyses } \\
\hline Renin (pg/ml) & $3.87(2.21 ; 6.66)$ & $4.59(2.43 ; 6.98)$ & 0.002 \\
\hline Interleukin-6 (pg/ml) & $2.16(2.06 ; 2.22)$ & $2.15(2.08 ; 2.22)$ & 0.286 \\
\hline C-reactive protein (mg/l) & $5.07(2.27 ; 26.6)$ & $4.12(2.99 ; 11.0)$ & 0.006 \\
\hline Glucose (mmol/l) & $5.15 \pm 0.92$ & $5.59 \pm 0.71$ & 0.001 \\
\hline Von Willebrand factor (\%) & $86.2(54.9 ; 135)$ & $60.1(40.9 ; 87.1)$ & $<0.001$ \\
\hline Triglycerides (mmol/l) & $1.18 \pm 1.03$ & $1.16 \pm 0.69$ & 0.882 \\
\hline Total cholesterol (mmol/l) & $4.48 \pm 1.17$ & $5.84 \pm 1.19$ & $<0.001$ \\
\hline \multicolumn{4}{|l|}{ Lifestyle } \\
\hline Y-Glutamyltransferase (U/l) & $36.7(16.6 ; 180)$ & $16.4(7.00 ; 76.0)$ & $<0.001$ \\
\hline Cotinine (ng/ml) & $2.58(2.00 ; 114)$ & $2.58(2.0 ; 245)$ & 0.669 \\
\hline Smoking (n) & 8 & 14 & 0.249 \\
\hline
\end{tabular}

Data expressed as mean \pm standard deviation, geometric mean (5th and 95th percentiles) and $n$

associated with percentage change in renin in the low renin black participants (model 1). In the same group, when we investigated whether the association of percentage change in renin with percentage change in PP is independent, percentage change in PP no longer entered the model (model 2). In the low renin white group the association of percentage change in renin with percentage change in IL-6 $(\beta=-0.24 ; p=0.005$; Table 3$)$ was also confirmed to be independent of various covariates. Power calculations indicated the population size to be sufficient.

In sensitivity analyses, we substituted percentage change in IL-6 with percentage change in CRP, the results remained the same in the low renin black group, but in the low renin white group only baseline renin $(\beta=-0.70 ; p<0.001)$ remained independently associated with percentage change in renin. When we substituted age with BMI in model 1, the results remained the same in both groups. After the addition of anti-inflammatory medication usage as covariate to the models the results also remained largely the same.

\section{Discussion}

The aim of the study was to investigate associations of change in renin with cardiovascular and inflammatory markers in a low renin bi-ethnic South African population. The most prominent result was an independent association between a decrease in renin levels and increased SBP in black participants with low renin levels. These findings are not only in line with previous findings indicating an association between low renin and blood pressure [22] but also strengthen the suggestion that low renin may be prospectively related to the early changes in the cardiovascular system before the onset of hypertension in black South Africans [23].

There are conflicting results in the literature about the different mechanisms leading to low renin levels in black participants when compared to white participants. These factors include high renal sodium reabsorption, [23, 24] reduced renin secretion rate [25] through decreased conversion of pro-renin to renin as a result of lower soluble plasma renin receptor found in blacks [26]. Other factors include polymorphisms in genes encoding for RAS proteins [23] and lifestyle factors such as rapid urbanization [23]. 
Table 2 Percentage change in anthropometric, cardiovascular, lifestyle and biochemical variables

\begin{tabular}{|c|c|c|c|}
\hline & \multicolumn{3}{|l|}{ Low renin } \\
\hline & Blacks $(n=66)$ & Whites $(n=71)$ & $p$-value \\
\hline Body mass index (\%) & $4.29(2.48 ; 6.10)$ & $3.35(1.60 ; 5.12)$ & 0.484 \\
\hline \multicolumn{4}{|l|}{ Cardiovascular measurements } \\
\hline Systolic blood pressure (\%) & $1.91(-0.21 ; 4.04)$ & $-1.77(-3.84 ; 0.30)$ & 0.020 \\
\hline Diastolic blood pressure (\%) & $2.53(0.29 ; 4.77)$ & $-0.62(-2.80 ; 1.56)$ & 0.059 \\
\hline Pulse pressure (\%) & $2.43(-1.09 ; 5.96)$ & $-2.52(-5.95 ; 0.91)$ & 0.057 \\
\hline Total peripheral resistance (\%) & $13.0(6.06 ; 19.99)$ & $2.23(-4.55 ; 9.01)$ & 0.036 \\
\hline Windkessel compliance (\%) & $-3.46(-7.68 ; 0.76)$ & $0.97(-3.14 ; 5.09)$ & 0.169 \\
\hline Carotid intima media thickness (\%) & $1.69(-0.73 ; 4.12)$ & $7.96(5.67 ; 10.24)$ & $<0.001$ \\
\hline \multicolumn{4}{|l|}{ Biochemical analyses } \\
\hline Renin (\%) & $-17.5(-24.3 ;-10.7)$ & $2.17(-4.39 ; 8.74)$ & $<0.001$ \\
\hline Interleukin-6 (\%) & $59.4(52.0 ; 66.9)$ & $35.5(28.3 ; 42.7)$ & $<0.001$ \\
\hline C-reactive protein (\%) & $0.34(-6.80 ; 7.48)$ & $-9.36(-16.3 ;-2.45)$ & 0.069 \\
\hline Glucose (\%) & $0.57(-4.73 ; 5.87)$ & $-22.7(-27.9 ;-17.6)$ & $<0.001$ \\
\hline Von Willebrand factor (\%) & $3.11(1.45 ; 4.77)$ & $5.76(4.14 ; 7.38)$ & 0.048 \\
\hline \multicolumn{4}{|l|}{ Lifestyle } \\
\hline ү-Glutamyltransferase (\%) & $-1.62(-6.73 ; 3.49)$ & $-4.17(-9.10 ; 0.75)$ & 0.512 \\
\hline Cotinine (\%) & $-1.80(-17.2 ; 13.6)$ & $6.86(-8.01 ; 21.7)$ & 0.443 \\
\hline
\end{tabular}

Data expressed as adjusted mean (least square mean) percentage change with $95 \%$ confidence intervals. Adjusted for baseline age, sex and baseline values

Although the exact mechanism linking decreased renin levels with increased blood pressure is not clear, it may be a result of volume loading. Volume loading is associated with increased sodium retention, which may result in the retention of water by the kidneys. When water is retained it may lead to expansion of the extracellular fluid volume and thereby increasing blood pressure [6]. In turn, this may lead to an even further suppression of renin secretion [6]. Our results, furthermore, suggest that the development of volume loading hypertension may be aggravated in the black population through excessive alcohol consumption [27] as indicated by the association of percentage change in renin with GGT levels, although not a focus of this article.

In the low renin white group, percentage change in renin was negatively associated with percentage change in IL-6 (inflammation). This is difficult to explain since activation of the RAS, through an increase in the rate limiting step, (renin) may lead to increased inflammation, since increased Ang II may stimulate the production of inflammatory cytokines in vascular smooth muscle and endothelial cells [28]. Inflammatory markers (IL-6 and CRP) are reliable biomarkers related to future risk for the development of CVD [19, 29]. Although
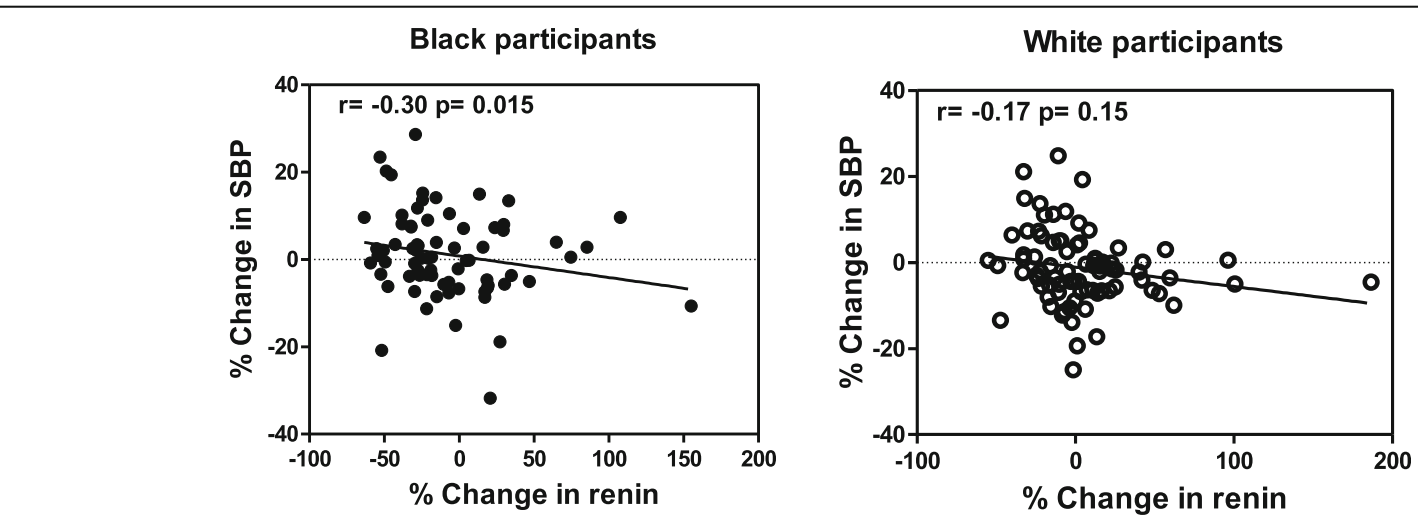

Fig. 1 Single regression analyses of percentage change in renin and percentage change in SBP in low renin black and white groups 
Table 3 Forward stepwise regression analysis with percentage change in renin as dependent variable in black and white participants

\begin{tabular}{|c|c|c|c|c|}
\hline \multirow{3}{*}{ Adjusted $R^{2}$} & \multicolumn{4}{|c|}{ Percentage change in renin } \\
\hline & \multicolumn{2}{|l|}{$\begin{array}{l}\text { Blacks }(n=76) \\
0.34\end{array}$} & \multicolumn{2}{|l|}{$\begin{array}{l}\text { Whites }(n=99) \\
0.54\end{array}$} \\
\hline & $\beta(95 \% \mathrm{Cl})$ & $p$-value & $\beta(95 \% \mathrm{Cl})$ & $p$-value \\
\hline Age (baseline) & $0.21(0.01 ; 0.41)$ & 0.046 & - & - \\
\hline Systolic blood pressure (\% change) & $-0.27(-0.48 ;-0.07)$ & 0.011 & - & - \\
\hline Gamma glutamyltransferase (\% change) & $0.26(0.05 ; 0.46)$ & 0.017 & - & - \\
\hline Renin (baseline) & $-0.46(-0.66 ;-0.25)$ & $<0.001$ & $-0.71(-0.87 ;-0.55)$ & $<0.001$ \\
\hline Interleukin-6 (\% change) & - & - & $-0.24(-0.40 ;-0.08)$ & 0.005 \\
\hline
\end{tabular}

Independent variables included in the model: percentage change in SBP, baseline SBP, age, percentage change in GGT, baseline GGT, baseline renin, percentage change in IL- 6 and baseline IL-6. -, Covariate did not enter model

there was a slight increase $(2 \%)$ in renin levels in the white group renin levels were still in the low range and may therefore not have been sufficient to elicit an increased inflammatory response.

Since there was no association between percentage change in renin levels and inflammatory markers in the low renin black group, despite the increase in inflammatory markers at follow-up. It is, therefore, expected in the black individuals that with decreased RAS activation, decreased secretion of inflammatory cytokines will ensue. However, in our study, mean IL-6 levels at baseline in the low renin black participants indicated high inflammatory levels. Our results suggest that other mechanisms may be involved in the stimulation of inflammatory responses, in this group. These mechanisms may include other enzymes such as chymase and catepsin which are involved in the conversion of Ang I to Ang II [30].

The results of this study have to be interpreted within the context of its strengths and limitations. This is a well-controlled study which included urbanized black and white participants, allowing comparison between these groups. This is, to the best of our knowledge, the first study investigating how changes in renin over a period of 3 years are associated with change in cardiovascular variables and inflammation in both black and white individuals. The limitation of this study is that our sample was relatively small and was recruited from urban areas in the Potchefstroom District of the NorthWest Province and cannot be seen as representative of the entire South African population. Although we investigated changes over time (3 year follow-up) our results are based on associations and we cannot infer causality. Another limitation is the unknown salt intake levels of these participants.

\section{Conclusion}

In the black participants with low renin levels at baseline, a further decrease in renin levels over 3 years is independently associated with an increase in SBP. These results strengthen the notion that low renin may be a causative factor in the development of hypertension in black South Africans, but that the renin- angiotensin system is not the driving force behind the increased inflammation observed in this low renin black group.

\section{Additional file}

Additional file 1: Table S1. Partial correlations of percentage change in renin with biochemical and cardiovascular variables. (DOCX 26 kb)

\section{Abbreviations}

$\% \Delta$ : Percentage change; Ang II: Angiotensin; CIMT: Carotid intima media thickness; CRP: C-reactive protein; CVD: Cardiovascular disease;

Cwk: Windkessel compliance; DBP: Diastolic blood pressure; GGT: Gamma glutamyl transferase; IL-6: Interleukin-6; SABPA: Sympathetic activity and Ambulatory Blood Pressure in Africans; SBP: Systolic blood pressure;

TPR: Total peripheral resistance; vWf: Von Willebrand Factor

\section{Acknowledgements}

The authors gratefully acknowledge every member and participant who took part in this study. We appreciate the technical assistance of Mrs. Tina Scholtz, Dr. Szabolcs Péter and Sr. Chrissie Lessing.

\section{Funding}

The North-West University, National Research Foundation (SA), Medical Research Council, Roche Products (Pty) Ltd., South Africa and Metabolic Syndrome Institute (France) was responsible for funding this project.

\section{Availability of data and materials}

The data supporting the findings of this study can be shared prior to publication of the manuscript.

\section{Authors' contributions}

RS was responsible for the planning, writing and composition of the manuscript. JMvR gave recommendations for the framework, writing and composition of the manuscript as well as the methodology. JMvR supervised the statistical analyses and helped with the formulation of the tables and figures. JmvR played an important part in gaining funding for this project. CMCM played a role in giving recommendations for the writing and composition of the manuscript as well as formulation of the tables and figures and conclusions. CMCM also supervised the statistical analyses. All authors read and approved the final manuscript.

\section{Competing interests}

The authors declare that they have no competing interests.

Consent for publication

Not applicable. 


\section{Ethics approval and consent to participate}

All the subjects were informed about the protocol of the study as well as the benefits and the risks involved in taking part, after which each participant signed an informed consent form. The study fulfilled all the requirements as stated in the Helsinki Declaration of 1975 (revised in 2004) for investigation on human subjects. The SABPA study was approved by the Ethics Review Board of the North-West University and this sub-study was also approved by the Health Research Ethics Committee of the North-West University.

\section{Publisher's Note}

Springer Nature remains neutral with regard to jurisdictional claims in published maps and institutional affiliations.

\section{Received: 9 December 2016 Accepted: 13 April 2017}

\section{Published online: 26 April 2017}

\section{References}

1. Day C, Gray A. Health and Related Indicators. (In Ntuli, A, Suleman, F, Barron, P, McCoy, D., eds). South African health review. Durban: Health system trust; 2001. p. 283-340.

2. Lloyd-Sherlock P, Beard J, Minicuci N, Ebrahim S, Chatterji S. Hypertension among older adults in low-and middle-income countries: prevalence, awareness and control. Int J Epidemiol. 2014;43(1):116-128.

3. Ferrario CM, Trask AJ, Jessup JA. Advances in biochemical and functional roles of angiotensin-converting enzyme 2 and angiotensin-(1-7) in regulation of cardiovascular function. Am J Phys Heart Circ Phys. 2005; 289(6):H2281-90

4. Suzuki Y, Ruiz-Ortega M, Lorenzo O, Ruperez M, Esteban V, Egido J. Inflammation and angiotensin II. Int J Biochem Cell Biol. 2003:35(6):881-900.

5. Navar LG, Harrison-Bernard LM, Imig JD, Wang C-T, Cervenka L, Mitchell KD. Intrarenal angiotensin II generation and renal effects of AT1 receptor blockade. J Am Soc Nephrol. 1999:10:S266-72.

6. Laragh $\mathrm{JH}$, Sealey JE. The plasma renin test reveals the contribution of body sodium-volume content $(\mathrm{V}$ ) and renin-angiotensin ( $\mathrm{R}$ ) vasoconstriction to long-term blood pressure. Am J Hypertens. 2011;24(11):1164-80.

7. He FJ, Markandu ND, Sagnella GA, MacGregor GA. Importance of the renin system in determining blood pressure fall with salt restriction in black and white hypertensives. Hypertension. 1998;32(5):820-4.

8. Gafane L, Schutte R, Van Rooyen J, Schutte A. Plasma renin and cardiovascular responses to the cold pressor test differ in black and white populations: the SABPA study. J Hum Hypertens. 2016;30:346-51.

9. Hamer M, Malan L, Schutte A, Huisman H, Van Rooyen J, Schutte R, Fourie C. Malan N, Seedat Y. Plasma renin responses to mental stress and carotid intima-media thickness in black Africans: the SABPA study. J Hum Hypertens. 2011;25(7):437-43.

10. van Rooyen JM, Schutte AE, Huisman HW, Schutte R, Fourie CM, Malan NT, Malan L. End-organ damage in urbanized Africans with low plasma renin levels: the SABPA study. Clin Exp Hypertens. 2014;36(1):70-5.

11. Schutte A, Van Vuuren D, Van Rooyen J, Huisman H, Schutte R, Malan L, Malan N. Inflammation, obesity and cardiovascular function in African and Caucasian women from South Africa: the POWIRS study. J Hum Hypertens. 2006:20(11):850-9.

12. Van der Walt C, Malan L, Uys AS, Malan NT. Low grade inflammation and ECG left ventricular hypertrophy in urban African males: the SABPA study. Heart, Lung and Circulation. 2013;22(11):924-9.

13. Malan L, Hamer M, Frasure-Smith N, Steyn HS, Malan NT. Cohort profile: sympathetic activity and ambulatory blood pressure in Africans (SABPA) prospective cohort study. Int J Epidemiol. 2015:44(6):1814-22.

14. Marfell-Jones MJ, Stewart A, de Ridder J: International standards for anthropometric assessment; 2012

15. Imholz BP, Wieling W, van Montfrans GA, Wesseling KH. Fifteen years experience with finger arterial pressure monitoring. Cardiovasc Res. 1998; 38(3):605-16.

16. Guelen I, Westerhof BE, van der Sar GL, van Montfrans GA, Kiemeneij F, Wesseling $\mathrm{KH}$, Bos WJW. Validation of brachial artery pressure reconstruction from finger arterial pressure. J Hypertens. 2008;26(7):1321-7.

17. Hietala J, Koivisto $H$, Anttila $P$, Niemelä O. Comparison of the combined marker GGT-CDT and the conventional laboratory markers of alcohol abuse in heavy drinkers, moderate drinkers and abstainers. Alcohol Alcohol. 2006;41(5):528-33.
18. Collaboration PS. Age-specific relevance of usual blood pressure to vascular mortality: a meta-analysis of individual data for one million adults in 61 prospective studies. Lancet. 2002;360(9349):1903-13.

19. Lindmark E, Diderholm E, Wallentin L, Siegbahn A. Relationship between interleukin 6 and mortality in patients with unstable coronary artery disease: effects of an early invasive or noninvasive strategy. JAMA. 2001;286(17):2107-13.

20. Imhof A, Froehlich M, Brenner $H$, Boeing H, Pepys MB, Koenig W. Effect of alcohol consumption on systemic markers of inflammation. Lancet. 2001; 357(9258):763-7.

21. Faul F, Erdfelder E, Lang A-G, Buchner A. G* power 3: a flexible statistical power analysis program for the social, behavioral, and biomedical sciences. Behav Res Methods. 2007;39(2):175-91.

22. He J, Klag MJ, Appel LJ, Charleston J, Whelton PK. The Renin-Rngiotensin system and blood pressure* differences between blacks and whites. Am J Hypertens. 1999;12(6):555-62.

23. Opie LH, Seedat YK. Hypertension in sub-Saharan African populations. Circulation. 2005;112(23):3562-8.

24. Bochud M, Staessen JA, Maillard M, Mazeko MJ, Kuznetsova T, Woodiwiss A, Richart T, Norton G, Thijs L, Elston R. Ethnic differences in proximal and distal tubular sodium reabsorption are heritable in black and white populations. J Hypertens. 2009;27(3):606.

25. Sagnella G. Why is plasma renin activity lower in populations of African origin? J Hum Hypertens. 2001;15(1):17-25.

26. Nguyen $G$, Blanchard A, Curis E, Bergerot D, Chambon Y, Hirose T, CaumontPrim A, Tabard SB, Baron S, Frank M. Plasma soluble (pro) renin receptor is independent of plasma renin, prorenin, and aldosterone concentrations but is affected by ethnicity. Hypertension. 2014;63(2):297-302.

27. Wilkinson S, Smith I, Williams R. Changes in plasma renin activity in cirrhosis: a reappraisal based on studies in 67 patients and" low-renin" cirrhosis. Hypertension. 1979;1(2):125-9.

28. Kranzhöfer R, Schmidt J, Pfeiffer CA, Hagl S, Libby P, Kübler W. Angiotensin induces inflammatory activation of human vascular smooth muscle cells. Arterioscler Thromb Vasc Biol. 1999;19(7):1623-9.

29. Yin $\mathrm{W}-\mathrm{H}$, Chen $\mathrm{J}-\mathrm{W}$, Jen $\mathrm{H}-\mathrm{L}$, Chiang $\mathrm{M}-\mathrm{C}$, Huang W-P, Feng A-N, Young MS, Lin S-J. Independent prognostic value of elevated high-sensitivity C-reactive protein in chronic heart failure. Am Heart J. 2004;147(5):931-8.

30. Barlucchi L, Leri A, Dostal DE, Fiordaliso F, Tada H, Hintze TH, Kajstura J, Nadal-Ginard B, Anversa P. Canine ventricular myocytes possess a reninangiotensin system that is upregulated with heart failure. Circ Res. 2001; 88(3):298-304

\section{Submit your next manuscript to BioMed Central and we will help you at every step:}

- We accept pre-submission inquiries

- Our selector tool helps you to find the most relevant journal

- We provide round the clock customer support

- Convenient online submission

- Thorough peer review

- Inclusion in PubMed and all major indexing services

- Maximum visibility for your research

Submit your manuscript at www.biomedcentral.com/submit
Biomed Central 